\title{
Voltage Stability Monitoring using Sensitivities Computed from Synchronized Phasor Measurement Data
}

\author{
Rujiroj Leelaruji, Student Member, IEEE, Luigi Vanfretti, Member, IEEE, and M. Shoaib Almas
}

\begin{abstract}
Wide-area early warning systems are dependent on synchrophasor data-based applications for providing timely information to operators so that preventive actions can be taken. This article proposes the use of voltage sensitivities computed from syncrhophasor data for voltage stability monitoring, and a visualization approach that can be implemented in wide-area early warning systems. In order to provide reliable information, synchrophasor data must be pre-processed to extract only the useful features embedded in measurements and correct for errors. Hence, this article also addresses the issue of data filtering and correction, and proposes a filtering methodology for robust voltage sensitivity computation. The methodology is developed considering both positive-sequence simulations for methodology development purposes, and real phasor measurement data from a real-time (RT) hardware-in-the-loop (HIL) laboratory for testing the robustness of the developed approach for use in the control room. The results from both approaches are contrasted against each other, the limitations of the positive-sequence simulation approach for developing PMU-data applications are highlighted and the challenges of working with the RT HIL lab are recognized.
\end{abstract}

Index Terms-Wide-Area Voltage Instability Detection, WideArea Monitoring Systems, Wide-Area Early Warning Systems, Visualization, PMU-based applications, Filtering

\section{INTRODUCTION}

$\mathbf{P}$ Hasor Measurement Units (PMUs) have been adopted to provide a high-sampling rate positive-sequence voltage and current phasors for Wide-Area Monitoring, Control and Protection (WAMPAC) Systems [1]. The successful deployment of PMUs [2] and applications that harvest their data in WAMPAC systems have the potential of gradually transforming today's power transmission networks into Smart Transmission Grids [3]. Within WAMPAC systems, widearea early warning systems are dependent on synchrophasor data-based applications for providing timely information to operators so that preventive actions can be taken.

As recognized in [3], the development and deployment of PMU data-based applications have been slow, limiting the potential of WAMPAC systems. Nevertheless, to date, a number of applications have been implemented in industrial Wide-Area Monitoring Systems (WAMS), mainly to provide awareness to disruptive events. The use of these applications is catered, for example, to provide the wide-area visibility [4], [5], the detection of critical oscillatory mode properties [6], [7], and the voltage instability detection [8]. Note that the design of

Manuscript submitted to the IEEE PES General Meeting 2012.

Invited Paper for the Panel Session: "Wide-Area Early Warning Systems", IEEE PES General Meeting 2012, San Diego, CA.

This work was supported in part by $\mathrm{EKC}^{2}$ - The Swedish Centre of Excellence in Electric Power Engineering.

L. Vanfretti is supported by the STandUP for Energy collaboration initiative and the KTH School of Electrical Engineering.

The authors are with the Electric Power Systems Division, School of Electrical Engineering, KTH Royal Institute of Technology, Teknikringen 33, SE-100 44, Stockholm, Sweden. E-mail: rujiroj@kth.se, luigiv@kth.se, msalmas@kth.se
WAMS and WAMPAC systems in general also includes the possibility for implementing other applications [9].

Most of the voltage instability detection methods that have been proposed, with two notable exceptions [10], [11], rely upon the construction of Thévenin equivalent models with synthesized parameters. Although useful and reliable, these methods have limitations which have been recognized in [11], [12] . With "wide-area methods" [10], [11], the use of sensitivities may have great potential for detecting the inception of voltage instabilities in wide-areas without depending on circuit synthesis. However, the computation of sensitivities from synchronized phasor-measurement data has several challenges.

First, the development of these methods has been traditionally carried out through positive-sequence-based models and simulations. This is reasonable in principle as the time scale of this type of instability is, in most cases, larger in comparison to other types of instabilities [13]. However, this has the disadvantage that the synthetic data generated by the simulation of these models does not contain the actual features that one expects from actual PMU data, nor do they deal with the possible data errors and inconsistencies embedded in synchrophasor streams.A lack of and/or incorrect data processing can yield incorrect information, which can mislead operators to a non-optimal preventive action, or even an improper corrective action that could lead to system collapse.

This article proposes the use of voltage sensitivities computed from syncrhophasor data for voltage stability monitoring, and a visualization approach that can be implemented in wide-area early warning systems. A filtering methodology for robust voltage sensitivity computation is proposed. The methodology is developed considering both positive-sequence simulations, and real phasor measurement data from a realtime (RT) hardware-in-the-loop (HIL) laboratory for testing the robustness of the developed approach in control room applications. The results from both approaches are contrasted against each other, the limitations of the positive-sequence simulation approach for developing PMU-data applications are highlighted and the challenges of working with the RT HIL lab are recognized.

The remainder of this article is structured as follows. Section II offers a synthesis of wide-area voltage stability monitoring concepts. Section III presents the experimental and simulation set-ups used for methodology development in this article, while Section IV briefly contrasts the difference between using standard simulations and PMU data. Section $\mathrm{V}$ describes the sensitivities considered, while Section VI describes the proposed filtering methodologies for pre-processing data for robust sensitivity calculations. Section VII proposes a visualization approach for monitoring voltage instabilities. Finally, in Section VIII, conclusions are drawn and future work is outlined. 


\section{Wide-Area Voltage Stability Monitoring CONCEPTS}

Voltage instability detection methods can be categorized in to the following branches: local [14], [15] versus widearea measurement [16] or synchornized [17] versus nonsynchornized [18] approaches. The idea behind these categories is on how the accumulated measurement information is used. To have an overall situation awareness, it appears that the entire power system state needs to be reconstructed from synchrophasor measurements and other data [19], or that different indices for different system components need to be computed to determine instabilities [20]. It can be argued that wide-area early warning systems could contain monitoring tools that display voltage stability indicators; however it is not clear how to present this information to operators so that it is easy to understand. Therefore, the ultimate goal of this article is to create the voltage stability indicators by computing sensitivities that are simple to comprehend.

As mentioned earlier, PMU data must be first pre-processed in order to compute accurate and robust sensitivities if they are to be used for control room applications. This issue is avoided in [10] by extracting the slope of the sensitivities instead of sensitivities itself. To achieve this, a parcel of data is needed, delaying the delivery of information to a widearea early warning system. The pre-processing issue is partly addressed in [12] by using filtering to separate the quasi-steady state components of simulated data; however, the approach is limited because the simulated data does not contain the correct features observed from PMU data and it is not capable to cater for inconsistencies and errors embedded in them. Hence, a filtering methodology for robust voltage sensitivity computation is proposed in Section VI.

\section{EXPERIMENTAL AND Simulation Set-UpS}

\section{A. Real-Time Hardware-In-Loop (RT HIL) Data Generation}

It is costly to adopt a real power system for an experimental purpose, and PMU data from utilities is not always available. Thus, to bridge the gap between theory and reality, a development and testing platform that performs as close as possible to real existing networks is adopted for the experiments involving real PMU data. This platform is available at SmarTS Lab, detailed information about this laboratory can be found in [21], a brief summary on how real-time data is generated is provided below.

Figure 1 shows the overall architecture of the SmarTS Lab, where PMUs and protection relays are connected in HardwareIn-Loop (HIL) fashion with a Real-Time (RT) simulator to acquire three-phase current and voltage waveforms from a test system which is simulated in real-time. The model of the test system was developed using MATLAB/ Simulink SimPowerSystems, and adapted using RT-Lab libraries for RT simulation. The synchrophasor data is streamed out from SEL PMUs through their Ethernet ports and received on a server which hosts a WAMPAC Application Host Platform. This platform includes a Phasor Data Concentrator (PDC) that time-aligns the synchrophasor input data from different PMUs

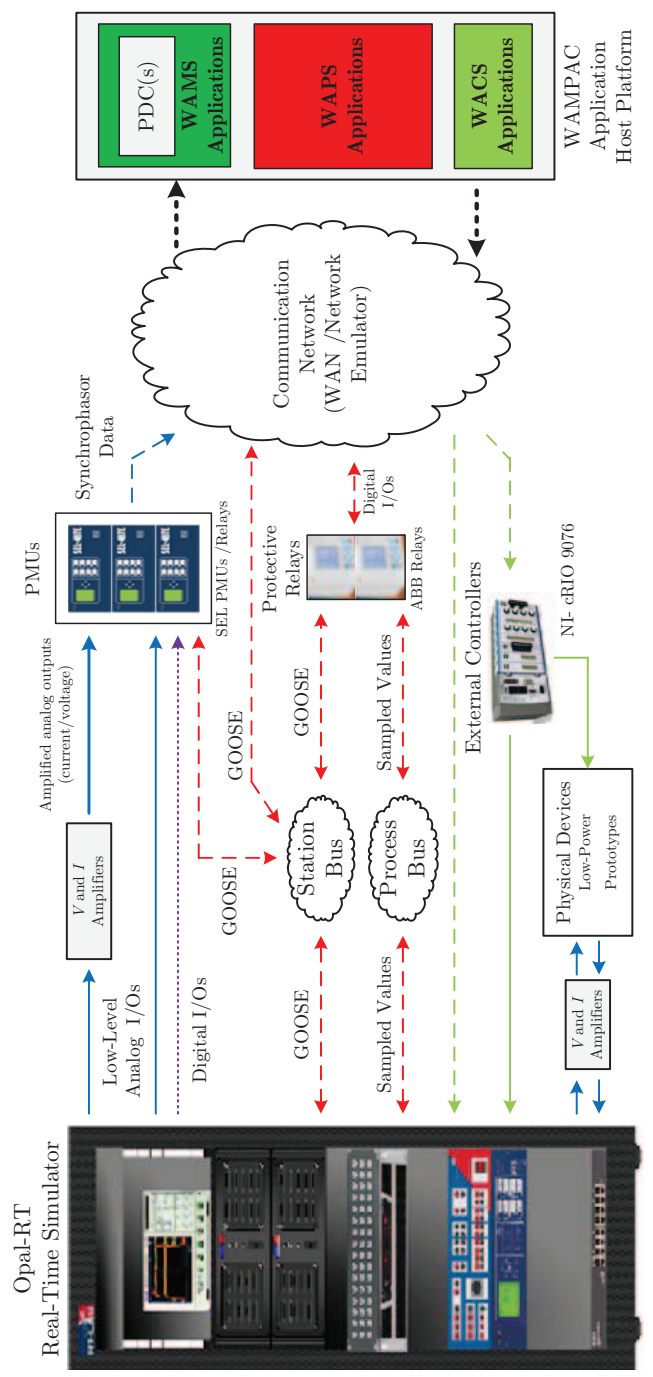

Fig. 1. General Architecture of SmarTS Lab for RT HIL Data Generation, see [21].

and broadcasts a single stream of data which is used for the experiments in this paper.

\section{B. Test System}

A one-line diagram of the test system is shown in Fig. 2. The system consists of a local area connected to a strong grid (Thevenin Equivalent) by two $380 \mathrm{kV}$ transmission lines. A motor load (rated 750 MVA, $15 \mathrm{kV}$ ) is connected at Bus 4 and supplied via a 380/15 ratio transformer. A load with constant power characteristics and load tap changer (LTC) dynamics at the distribution side are explicitly modelled at Bus 5. A local generator (rated $450 \mathrm{MVA}, 20 \mathrm{kV}$ ) equipped with a simplified IEEE ST1A excitation system [22], a standard speed-governing model and a simplified linear model of steam turbine (which can be found in an IEEE Working Grouping Report [23]) is connected at Bus 2 to supply the loads through a 20/380 ratio transformer. Moreover, the load at Bus 5 is modelled as constant power. This load is also equipped with an automatic on-load tap changer (OLTC) where changing logic at a given time instant can be found in [24].

This test system is modelled both with the DIgSILENT PowerFactory simulation software and in the RT simulator. 


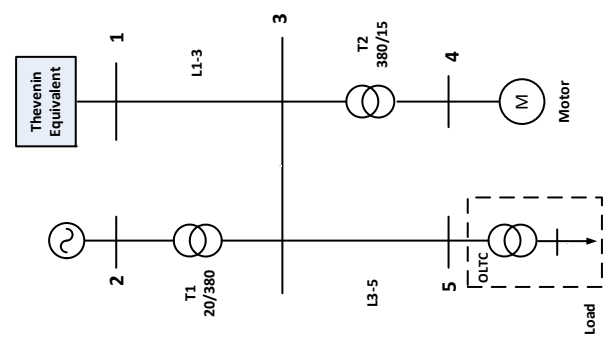

Fig. 2. Test system used for generating voltage instability scenarios

\section{PSB Simulations Vs. "REAL" PMU Data}

The reason of having the test system in two test platforms (positive-sequence-based (PSB) simulations and RT-HIL) is to demonstrate that the development of a monitoring tool cannot rely only on simulation results. This is because the PSB simulation-based PMU applications may not give satisfactory results when being exposed to "real-data". PSB simulations may exclude the effect of switching devices and events on measurements, ambient behavior, noise and outliers in actual PMU data.

The voltage instability scenario used for demonstration is implemented by increasing the load at Bus 5 at $t=70 \mathrm{~s}$ of the test system shown in Fig. 2. Typical constant active and reactive power models are used, the load is assumed to change as follows:

$$
\begin{aligned}
P_{L} & =P_{L o}(1+\lambda) \\
Q_{L} & =Q_{L o}(1+\lambda)
\end{aligned}
$$

where $P_{L o}$ and $Q_{L o}$ are the initial base active and reactive powers, respectively, and $\lambda$ is a varying parameter representing the loading factor. The on-load tap changer (OLTC) tries to restore the voltage at the load bus within its deadband [24]. Since the load increases monotonically, the OLTC unsuccessfully attempts to restore the load bus voltage, until it reaches its lower limit. The load bus voltage then decreases stepwise accordingly and the system collapses at $t=440 \mathrm{~s}$ (see Fig. 3).

The same experiment is conducted in the laboratory using the RT HIL approach, and the syncrhophasors for Bus 5 are acquired. Fig. 4 shows the voltage magnitude from the phasor streamed out from a SEL PMU. Note that the traces obtained from the PMU have a higher resolution than those from the PSB simulation (Fig. 3). Notice that Fig. 4 clearly contains not only electromechanical oscillations, but also noise, and outliers due to the switching of discrete devices whereas Fig. 3 does not contain most of these characteristics. To develop a robust wide-area monitoring application, these data features need to be considered.

\section{PV AND QV SEnsitivities AS VS IndiCATORS}

The use of voltage sensitivities for voltage instability detection has been proposed in [11], [25], [26], [10]. In this study, the voltage sensitivities used are derived by using only the power flow in the line (one direction) instead of injected power flow of the bus (summation of power flow) to reconstruct the Jacobian matrix. Note that differently from [11], [12], the network parameters are not needed, and the calculations are solely based on PMU data.

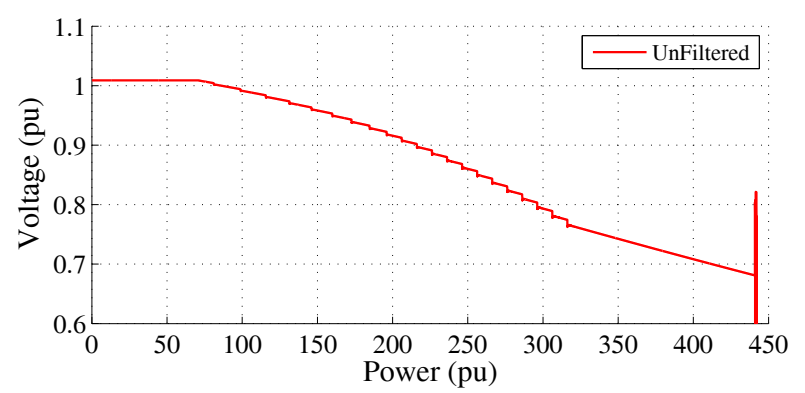

Fig. 3. Voltage at Bus 5 from a DIgSILENT PowerFactory PSB Simulation

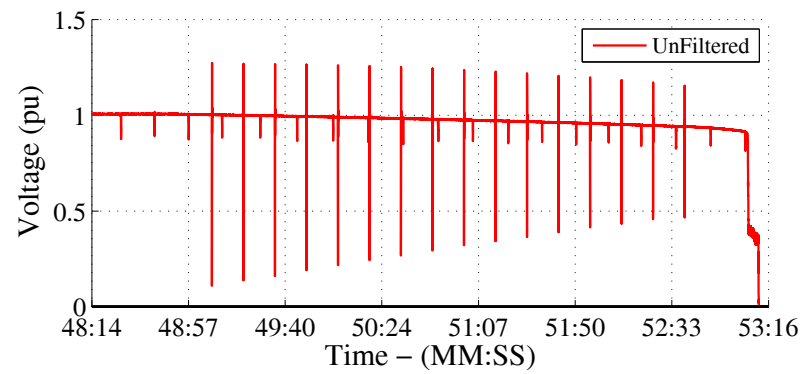

(a) Voltage Magnitude at Bus 5 - complete time span until collapse.

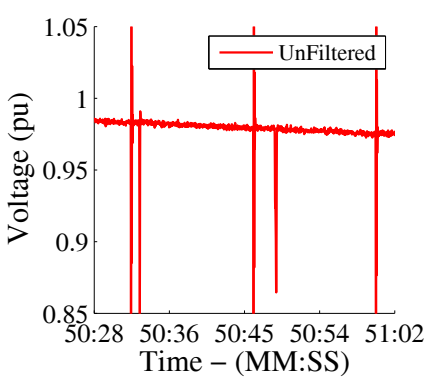

(b) Zoom of Fig. 4a

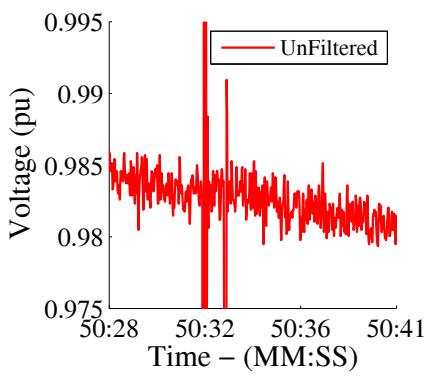

(c) Zoom of Fig. 4b
Fig. 4. Voltage Magnitude at Bus 5 from PMU Measurements.

The transmitted power on the line can be expressed as follows:

$$
\begin{aligned}
\bar{S}_{i k} & =V_{i} e^{j \delta_{i}}\left\{V_{i} e^{j \delta_{i}} y_{i k 0}+\left(V_{i} e^{j \delta_{i}}-V_{k} e^{j \delta_{k}}\right) y_{i k}\right\}^{*} \\
P_{i k} & =\operatorname{Re}\left(\bar{S}_{i k}\right), \quad Q_{i k}=\operatorname{Im}\left(\bar{S}_{i k}\right)
\end{aligned}
$$

where

$V_{i}=$ voltage magnitude at Bus $i$.

$\delta_{i}=$ voltage angle at Bus $i$.

$y_{i k 0}=$ half of the shunt capacitance of the line.

$y_{i k}=\frac{1}{z_{i k}}$ where $z_{i k}=$ line impedance.

$\bar{S}_{i k}=$ complex power transmitted from Bus $i$ to Bus $k$.

$P_{i k}=$ transmitted real power from Bus $i$ to Bus $k$.

$Q_{i k}=$ transmitted reactive power from Bus $i$ to Bus $k$.

$\delta_{i k}=\delta_{i}-\delta_{k}$.

From (3) it follows that to calculate the real and reactive power flows through the transmission lines, the magnitude and angle of the voltage must be known at both ends. 


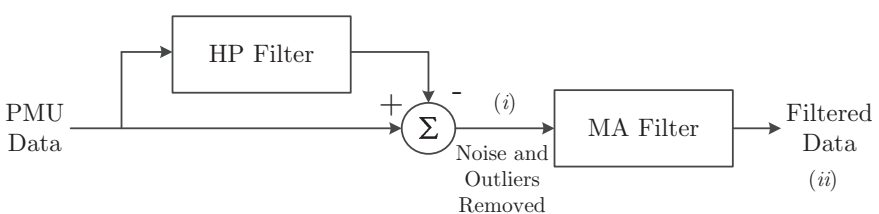

Fig. 5. Proposed filtering approach for pre-processing data to compute voltage stability indicators.

The modified Jacobian matrix can be constructed as follows:

$$
J(\delta, V)=\left[\begin{array}{cc}
P_{i k_{\delta}} & P_{i k_{V}} \\
Q_{i k_{\delta}} & Q_{i k_{V}}
\end{array}\right]
$$

where

$P_{i k_{\delta}}=P_{i k}(t)-P_{i k}(t-1) / \delta(t)-\delta(t-1)$

$P_{i k_{V}}=P_{i k}(t)-P_{i k}(t-1) / V(t)-V(t-1)$

Similar expressions can also be derived for $Q_{\delta}$ and $Q_{V}$.

Finally, sensitivities can be found from the inverse of (4), however, this inverse is not explicit calculated, instead each element of the inverse is obtained from the PMU data. The sensitivity values are consistent with the assumption of steadystate operation, however their value will increase when a system is stressed, hence they can be used as voltage instability indicators. The value increases abruptly to very high positive and switches from positive to negative in case of lack of reactive power support for $d V_{i} / d Q_{i k}$ or when the maximum power transfer is reached for $d V_{i} / d P_{i k}$. A graphical example is illustrated in Section VI.

\section{Vi. Filtering Methodology}

The proposed filtering method used in this study is similar that in [27]. Its aim is to smooth all signals before computing voltage sensitivities. Figure 5 shows a block diagram of the processing method. It starts by taking raw data and applying a high pass filter with cutoff frequency of $1.37 \mathrm{~Hz}$. This cutoff frequency corresponds to the dominant oscillatory component in the system. Then, the original data is subtracted by the filtered data. By applying this subtraction, electromechanical oscillations and large outliers are removed from the original signal, this is shown in Fig. 5 with a $(i)$; signals from this step are termed "filtered" signals. An additional step is to apply a moving average (MA) filter by computing the mean and standard deviation of the filtered signal. As denoted in Fig. 5, these signals are termed "filtered $+M A$ ". The size of the MA window is designed to cover the data which deviates from the mean value by three standard deviations of the filtered signal. The MA aids to increase the robustness of the computed sensitivities. This filtering method is applied to both the DIgSILENT PowerFactory simulation (of $0.001 \mathrm{sec}$ stepsize) and the PMU measurement data (50 samples per second).

Figure 6 and 7 show the difference between filtering and not filtering voltages and the corresponding PV-curve at Bus 5 , respectively. Figure 8 depicts the plot of voltage stability sensitivity $(\mathrm{dV} / \mathrm{dP})$ at Bus 5 that obtained by using data from PSB simulation.

As mentioned in Section $\mathrm{V}$, the sensitivity $\left(d V_{i} / d P_{i k}\right.$ in this case) can be used to generate an early signal warning when its value changes from positive to negative; this occurs at $t=400 \mathrm{~s}$ (while system collapse at $t=440 \mathrm{~s}$ ). This early warning signal can be generated by setting a threshold value (e.g., $d V_{5} / d P_{53}=0.08$ ); this allows detection before the sensitivity changes abruptly to a large positive value. This can be verified from Fig. 6. The spikes shown in the green in Fig. 8 correspond to OLTC tap position changes. It can also be noted that the sensitivity calculated from the unfiltered data (green dashed line) are vulnerable to OLTC tap switching and vary abruptly compared with those computed using data which has been filtered, or filtered with MA. Figure 9 shows that the

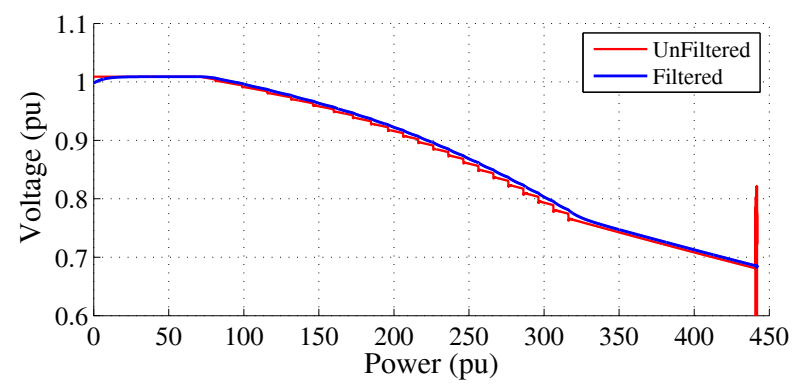

Fig. 6. Filtered and Unfiltered Voltage at Bus 5 (PSB Simulation)

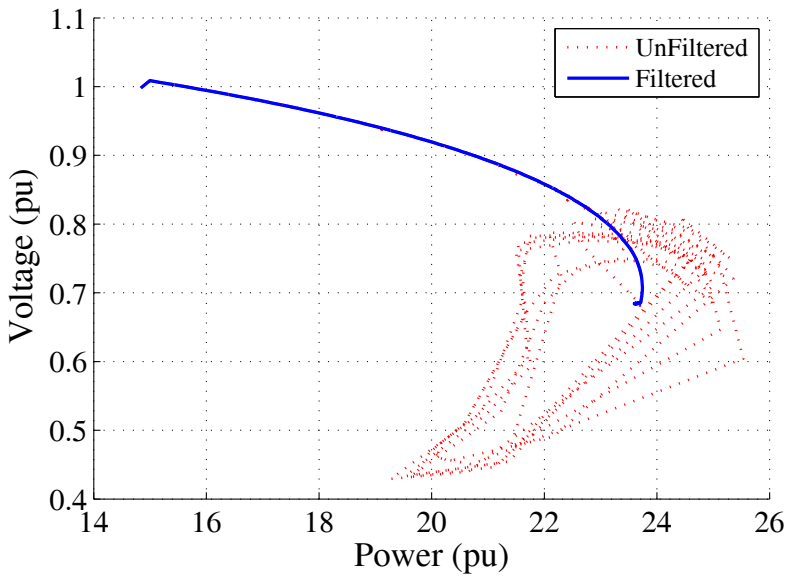

Fig. 7. Filtered and Unfiltered PV-curve (PSB Simulation)

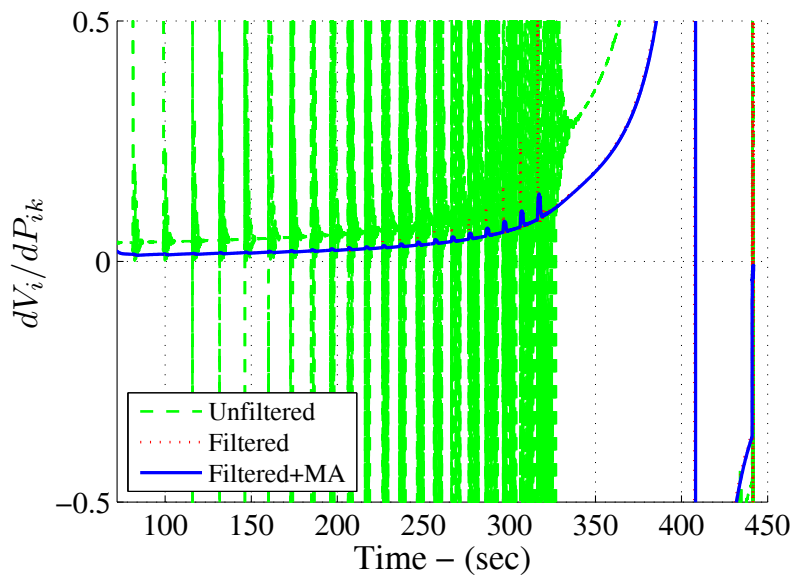

Fig. 8. Plot of calculated $d V_{5} / d P_{53}$ sensitivity at Bus 5 from filtered PSB simulation data. 


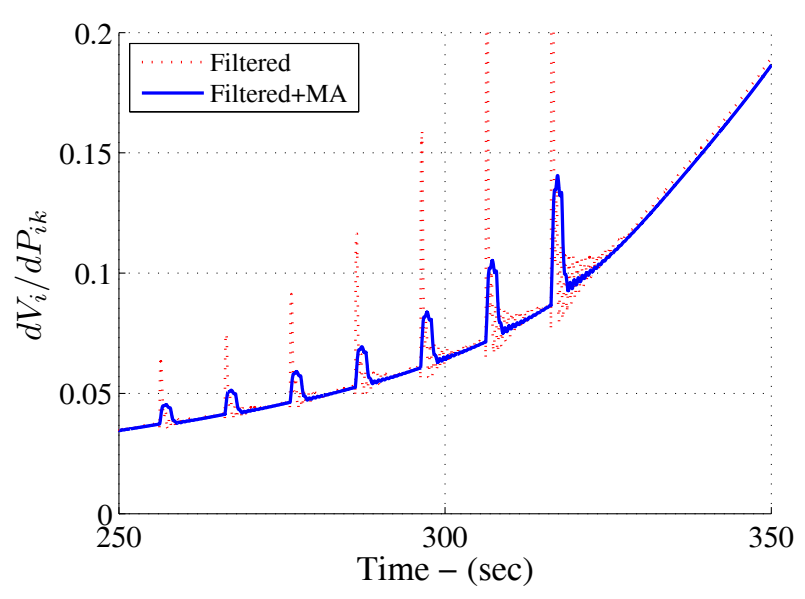

Fig. 9. Zoom of Fig. 8

filtered data with MA approach provides a more robust result compared to the one without MA. However, these filtering methods (either with or without moving average window) introduce a small delay to the calculation $(\approx 5 \mathrm{sec}$ for this simulation). Similar results were obtained using PMU data, which are shown in Fig. 10a. Here the spikes due to OLTC switching that appear in the computed sensitivities are much higher than those obtained using PSB simulation results. One of the reasons is than the test system running in RT HIL is more detailed that the one in the PSB simulator. In addition, there are other nuisances in the PMU data generated from the RT HIL simulator such as outliers and noise.

\section{Visualization APPROACH}

For the visualization of voltage stability indicators computed from sensitivities, the voltage phasors are gathered from different locations of the system shown in Fig. 2. Each incoming voltage phasor from different locations are filtered as described in Section VI. Then, the active and reactive power flow of each line are calculated from (3), consequently sensitivities are computed as in (4). The computed sensitivities for each location are assembled together in one display which maps their values to a contour overlaying the power system oneline diagram. Figure 11 shows the voltage stability monitor screen-shot of $d V_{5} / d P_{53}$. As shown in Fig. 11, the contour is on the left-hand side while the sensitivities are displayed on the top-right-hand side; a vertical red line indicates the time instant for which the contour is being computed. The bottomright-hand side is the PV-curve of a particular bus chosen by the user. A red circle indicates the current operating condition, which has been computed from the PMU data. Vertical and horizontal yellow and red dash-dot lines indicate the distance to the maximum power transfer (active power margin) for the $d V_{i} / d P_{i k}$ thresholds that have been set by user. Determination of the $\mathrm{PV}$ and $\mathrm{QV}$ curves and $P \& Q$ margins for each bus can be carried out off-line through continuation studies, or on-line as proposed in [28]. All of these quantities can be updated dynamically as the data is processed and passed to the display for visualization. Two thresholds for alarms are set at $d V_{5} / d P_{53}=0.08$, for an early warning signal (yellow

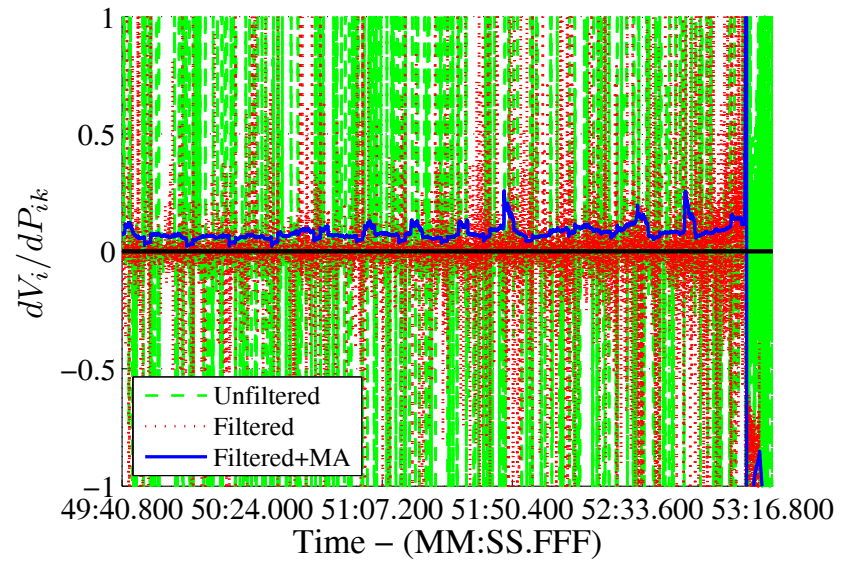

(a) Plot of calculated $d V_{5} / d P_{53}$ sensitivity at Bus 5 using PMU data.

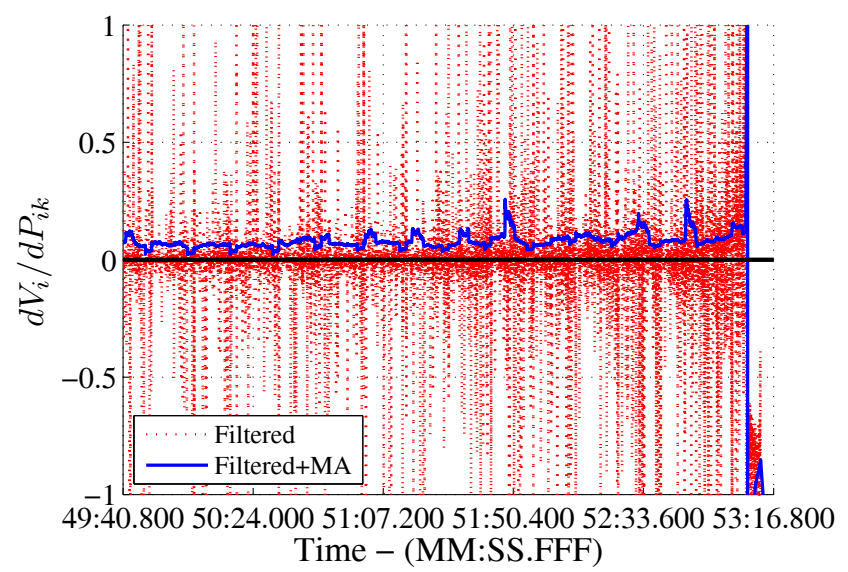

(b) Only filtered and filtered + MA results of Fig. 10a

Fig. 10. $d V_{5} / d P_{53}$ sensitivity at Bus 5 using PMU data generated from the RT HIL experiment.

dash-dot line), and $d V_{5} / d P_{53}=0.24$, for a final alarm (red dash-dot line). A similar screenshot of $d V_{5} / d Q_{53}$ is shown in Fig. 12.

To explain how this wide-area early warning monitoring tool works, two screenshots at two different times (I at $t=240 \mathrm{~s}$ and II at $t=360 \mathrm{~s}$ ) are captured and combined as shown in Fig. 13. The voltage instability scenario is implemented by increasing load at Bus 5 as explained earlier. The load increase rises the value of the sensitivities, this in turn changes the colors of the contour map. This color corresponds to the sensitivities value. The selected QV-curve (of Bus 5, in this case) changes its value decreasingly when the load increases. Before the system collapses, an early warning signal can be generated by setting different threshold values. A warning signal can be generated when $d V_{5} / d Q_{53}=0.2$ or a final alarm signal in case $d V_{5} / d Q_{53}=0.6$.

\section{CONCLUSIONS AND FUtURE WORK}

This article has described an approach for using voltage sensitivities computed from synchrophasor data, and a visualization approach that can be implemented for wide-area voltage stability monitoring to be used in wide-area early warning systems. If implemented for control room use, this 
Voltage Stability Indicator Contour: dV/dP Sesitivities Computed through Filter + Moving Average

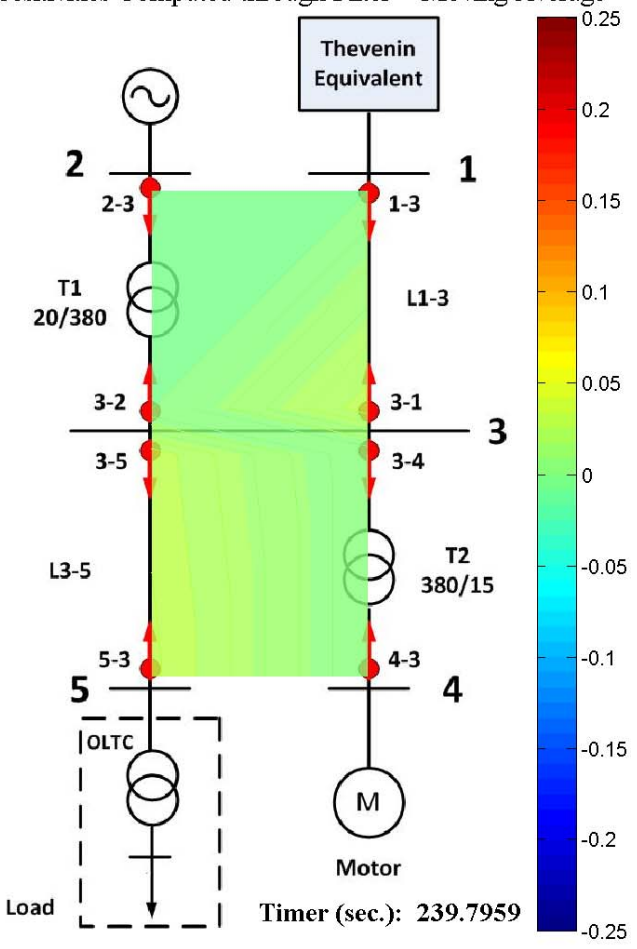

Fig. 11. Screenshot of Voltage Stability Monitor: $d V_{5} / d P_{53}$ Sensitivities

Voltage Stability Indicator Contour:

dV/dQ Sesitivities Computed through Filter + Moving Average

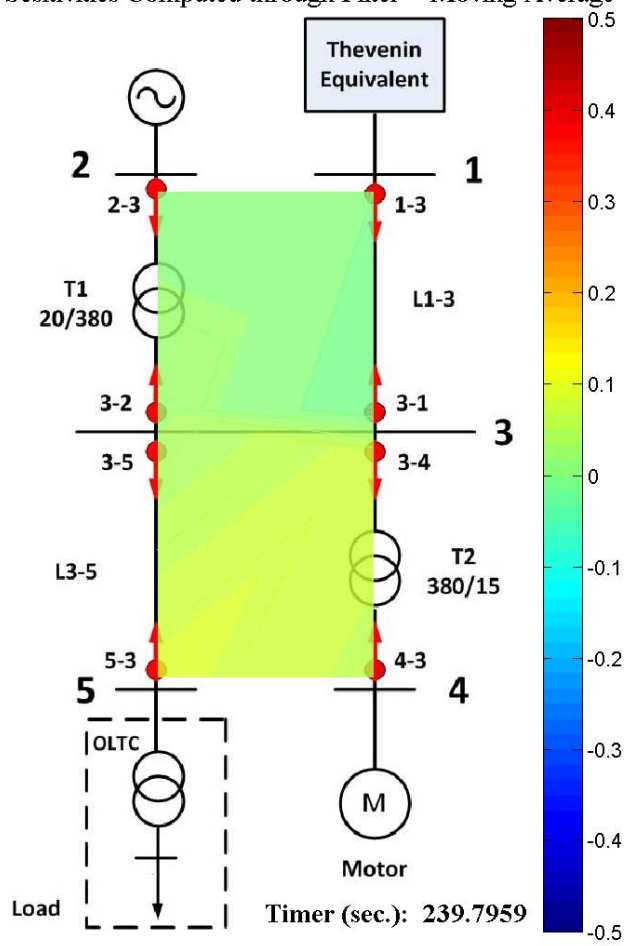

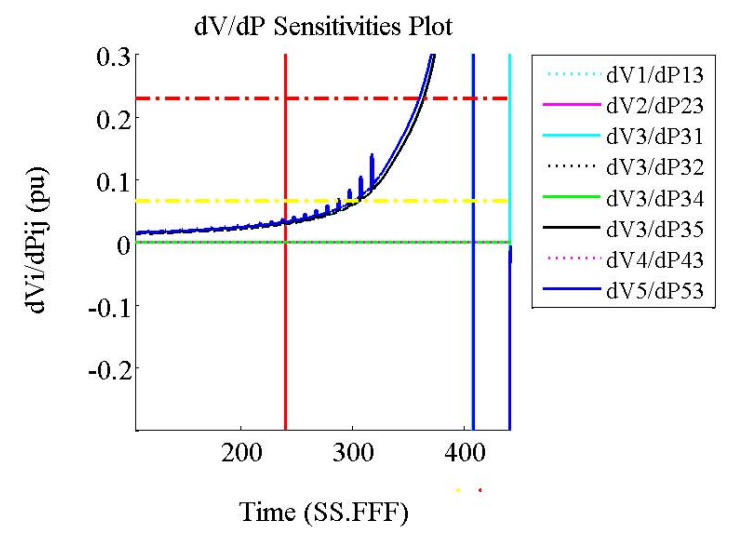

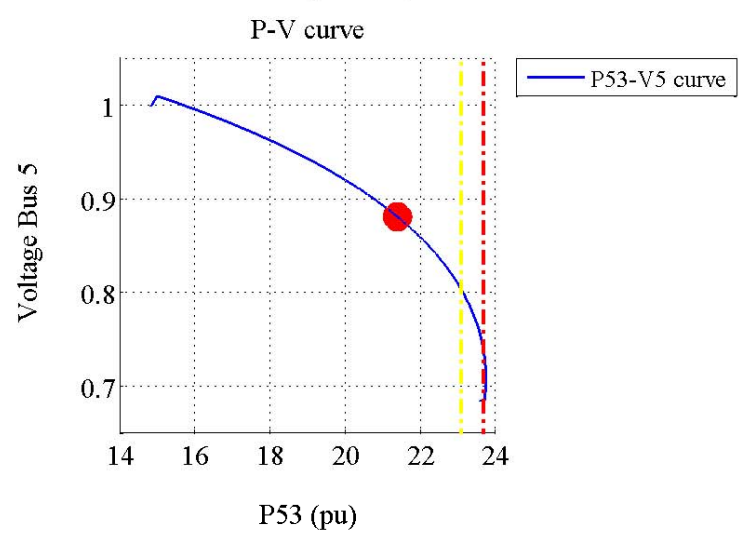

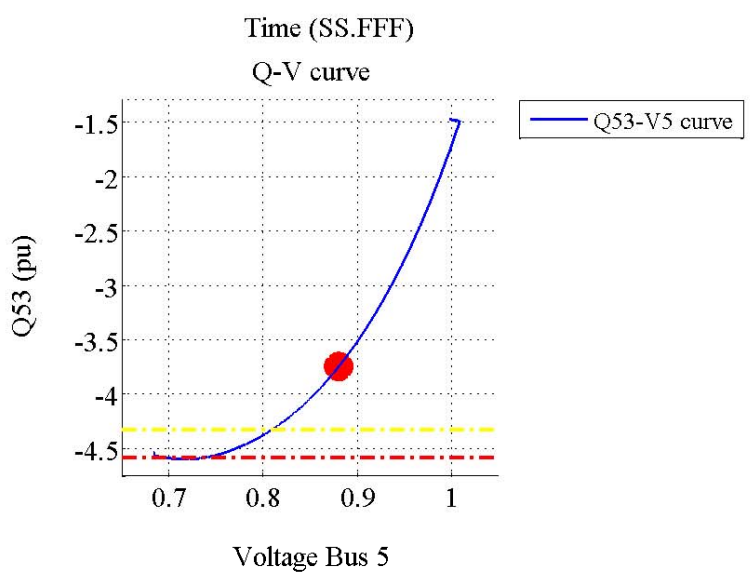

Fig. 12. Screenshot of Voltage Stability Monitor: $d V_{5} / d Q_{53}$ Sensitivities 


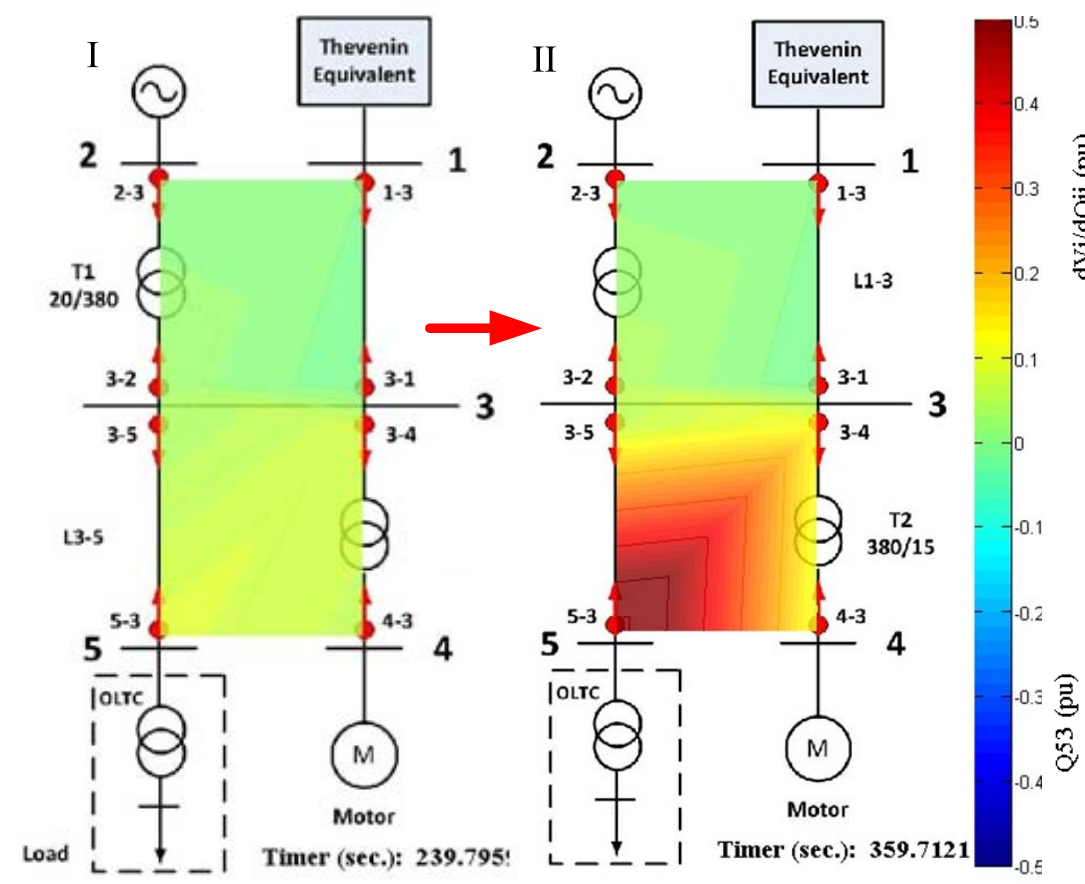

Fig. 13. Transition of $d V_{5} / d Q_{53}$ sensitivities from $t=240 \mathrm{~s}$ to $t=360 \mathrm{~s}$

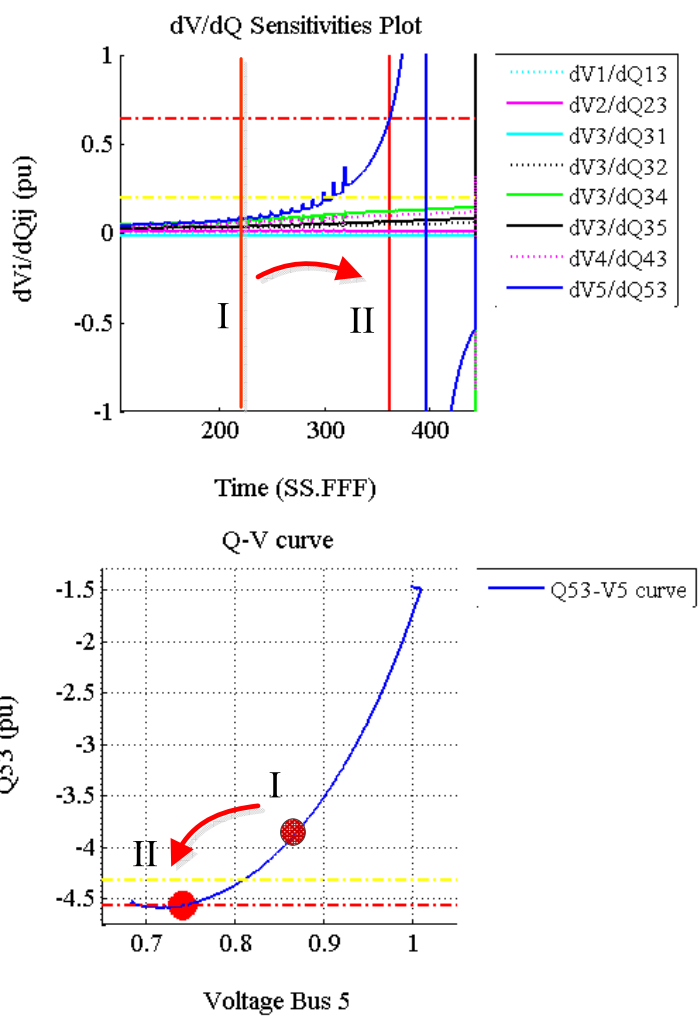

monitoring tool can be used by system operators in order to track the states of power system during normal and severe operating conditions.

The idea behind this approach is to ease system operators' tasks by automatically generating early warning signals before system collapse. To achieve this, sensitivities calculated from voltage phasors are utilized as stability indicators. Different system conditions are determined by the sensitivities which need to be computed from filtered synchrophasor measurement data. The filtering approach has a large impact in sorting out unwanted electromechanical oscillations, noise and outliers before computing the sensitivities. Both visualization and filtering approaches are validated by using positive-sequence simulation and PMU data from a real-time hardware-in-theloop laboratory.

The most important lesson to share from this experience is that any PMU-data application for WAMS, and especially early warning systems, must be thoroughly tested with actual PMU measurements to guarantee its robustness. The effects of inherent data characteristics will require algorithms to cater for relevant features which are simply not present from traditional positive-sequence simulations. To this end the use of a realtime hardware-in-the-loop laboratory has been shown to be very advantageous.

There are several issues that must be further addressed, including but not limited to: optimal filter design for sensitivity computations, base-lining for determining thresholds for different voltage instability indicators. The ultimate goal is not only to have real-time visualization of power systems and early warnings, but also to automate existing controllers in the power system with a control algorithm that acts before the PMU data is centralized in the control room.

\section{REFERENCES}

[1] V. Terzija, G. Valverde, D. Cai, P. Regulski, V. Madani, J. Fitch, S. Skok, M. Begovic, and A. Phadke, "Wide-Area Monitoring, Protection, and Control of Future Electric Power Networks," Proceedings of the IEEE, vol. 99, no. 1, pp. $80-93$, jan. 2011.

[2] A. Phadke and R. de Moraes, "The Wide World of Wide-area Measurement," IEEE Power and Energy Magazine, vol. 6, no. 5, pp. 52 -65, september-october 2008.

[3] A. Bose, "Smart Transmission Grid Applications and Their Supporting Infrastructure," IEEE Transactions on Smart Grid, vol. 1, no. 1, pp. 11-19, June 2010.

[4] Y. Zhang, P. Markham, T. Xia, L. Chen, Y. Ye, Z. Wu, Z. Yuan, L. Wang, J. Bank, J. Burgett, R. Conners, and Y. Liu, "Wide-Area Frequency Monitoring Network (FNET) Architecture and Applications," IEEE Transactions on Smart Grid, vol. 1, no. 2, pp. 159-167, Sept 2010.

[5] J. Zuo, R. Carroll, P. Trachian, J. Dong, S. Affare, B. Rogers, L. Beard, and Y. Liu, "Development of TVA SuperPDC: Phasor applications, tools, and event replay," in Power and Energy Society General Meeting Conversion and Delivery of Electrical Energy in the 21st Century, 2008 IEEE, July 2008, pp. 1-8.

[6] A. Hauer, D. Trudnowski, and J. DeSteese, "A Perspective on WAMS Analysis Tools for Tracking of Oscillatory Dynamics," in IEEE Power Engineering Society General Meeting, 2007, pp. 1-10, dOI: 10.1109/PES.2007.386186.

[7] D. Trudnowski, J. Pierre, N. Zhou, J. Hauer, and M. Parashar, "Performance of Three Mode-Meter Block-Processing Algorithms for Automated Dynamic Stability Assessment," IEEE Transactions on Power Systems, vol. 23, no. 2, pp. 680-690, May 2008.

[8] M. Parashar and J. Mo, "Real Time Dynamics Monitoring System (RTDMS): Phasor Applications for the Control Room," in 42nd Hawaii International Conference on System Sciences, 2009 (HICSS '09), Jan 2009.

[9] J. Hauer and J. DeSteese, "Descriptive Model of Generic WAMS," Pacific Northwest National Laboratory,Richland, WA., Tech. Rep., 2007.

[10] Venkatasubramanian, V. and Xing Liu and Guoping Liu and Qiang Zhang and Sherwood, M., "Overview of wide-area stability monitoring algorithms in power systems using synchrophasors," in American Control Conference (ACC), 2011, 29 2011-july 1 2011, pp. $4172-4176$.

[11] M. Glavic and T. Van Cutsem, "Wide-Area Detection of Voltage Instability From Synchronized Phasor Measurements. Part I: Principle," IEEE Transactions on Power Systems, vol. 24, pp. 1408 - 1416, 2009. 
[12] M. Glavic, M and T. Van Cutsem,, "Wide-Area Detection of Voltage Instability From Synchronized Phasor Measurements. Part II: Simulation Results," IEEE Transactions on Power Systems, vol. 24, pp. 1417 - 1425, 2009.

[13] C. Taylor, Power System Voltage Stability, N. Balu and D. Maratukulam, Eds. McGraw-Hill, 1994.

[14] K. Vu, M. Begovic, D. Novosel, and M. Saha, "Use of local measurements to estimate voltage-stability margin," IEEE Transactions on Power Systems, vol. 14, pp. 1029 - 1035, 1999.

[15] I. Smon, G. Verbic, and F. Gubina, "Local Voltage-Stability Index Using Tellegen's Theorem," IEEE Transactions on Power Systems, vol. 21, pp. $1267-1275,2006$

[16] C. D. Vournas and N. G. Sakellaridis, "Tracking Maximum Loadability Conditions in Power Systems," in 2007 iREP Symposium Bulk Power System Dynamics and Control - VII. Revitalizing Operational Reliability, 2007.

[17] B. Milosevic and M. Begovic, "Voltage-stability protection and control using a wide-area network of phasor measurements," IEEE Transactions on Power Systems, vol. 18, pp. 121-127, 2003.

[18] L. Bao, Z. Huang, and W. Xu, "Online Voltage Stability Monitoring Using Var Reserves," IEEE Transactions on Power Systems, vol. 18, pp. 1461-1469, 2003.

[19] M. Glavic and T. Van Cutsem, "Investigating state reconstruction from scarce synchronized phasor measurements," in 2011 IEEE Trondheim PowerTech, 2011.

[20] B. Milosevic and M. Begovic, "Voltage Stability Protection and Control using a Wide-Area Network of Phasor Measurements," IEEE Transactions on Power Systems, vol. 18, pp. 121-127, 2003.

[21] L. Vanfretti et al, "SmarTS Lab - A Laboratory for Developing Applications for WAMPAC Systems," in submitted for publication, IEEE PES General Meeting 2012, july 2012.

[22] R. Leelaruji and L. Vanfretti, “'All-in-one' test system modelling and simulation for multiple instability scenarios," KTH, Royal Institute of Technology, Internal Report, April 2011.

[23] IEEE Committee Report, "Dynamic Models for Steam and Hydro Turbines in Power System Studies," IEEE Transactions on Power Apparatus and Systems, vol. PAS-92, pp. 1904-1915, 1973.

[24] T. Van Cutsem and C. Vournas, Voltage Stability of Electric Power Systems. Kluwer Academic Publishers, 1998.

[25] T. Van Cutsem, "An approach to corrective control of voltage instability using simulation and sensitivity," IEEE Transactions on Power Systems, vol. 2, pp. 616-622, 1995.

[26] M. Begovic and A. G. Phadke, "Control of voltage stability using sensitivity analysis," IEEE Transactions on Power Systems, vol. 7, pp. 114-123, 1992.

[27] L. Vanfretti, J. H. Chow, U. Aliyu, L. Dosiek, J.Pierre, D. Trudnowski, and R. Garcia-Valle, "Estimation of the Nigerian Power System Electromechanical Modes using FDR Measurements," in 9th International Conference on Power System Operations and Planning (ICPSOP), 2010.

[28] M. Parniani, J. Chow, L. Vanfretti, B. Bhargava, and A. Salazar, "Voltage Stability Analysis of a Multiple-Infeed Load Center Using Phasor Measurement Data," in 2006 IEEE PES Power Systems Conference and Exposition, 2006 (PSCE '06), Nov 2006, pp. 1299 -1305.
Rujiroj Leelaruji received the B.Sc. degree in electrical engineering from Sirindhorn International Institute of Technology (SIIT), Thailand, in 2004, and the M.Sc. degree in electric power engineering from KTH Royal Institute of technology, Stockholm, Sweden, in 2007. He is currently a Ph.D. student within the Electric Power Systems (EPS) Division at KTH. The main theme of his current research is the development and implementation of an algorithm for coordinating system protections and HVDC/FACTS controllers that can result in preventive measures mitigating wide-area system collapses.

Luigi Vanfretti (Student Member '03, M'10) is an Assistant Professor at the Electric Power Systems Division, School of Electrical Engineering, KTH Royal Institute of Technology, Stockholm, Sweden. He received the Electrical Engineering Degree from Universidad de San Carlos de Guatemala in June 2005. He was a visiting researcher at the Department of Electronics and Electrical Engineering of The University of Glasgow, Scotland in 2005. He received his M.Sc. in 2007 and Ph.D. in 2009, both in Electric Power Engineering, from Rensselaer Polytechnic Institute (RPI), Troy, NY, USA. For his research and teaching work towards his Ph.D. he was awarded the Charles M. Close Award from Rensselaer Polytechnic Institute.

Since 2009, he has served as Secretary of the IEEE Task Force on Open Source Software and as WG Organizer for the IEEE PSDP Working Group on Power System Dynamic Measurements. His research interests are in the general area of modeling, dynamics, stability and control of power systems; while his main focus is on the development of applications of PMU data. He is an evangelist of Free/Libre and Open Source Software for power system engineering education.

Shoaib Almas has recently completed his M.Sc. in Electric Power Engineering from KTH Royal Institute of Technology, Stockholm Sweden. Previously he did his B.Sc. in Electrical Engineering from NUST, Pakistan. He has a professional experience in substation automation and protection. Currently he is working at the SmarTS Laboratory at KTH 